\title{
Note
}

\section{A Numerical Method for the Self-Similar Hypersonic Viscous Shear Layer}

\section{INTRODUCTION}

In laminar fluid motion at high Reynolds numbers, viscous effects are important primarily in thin layers described by the boundary-layer approximation to the Navier-Stokes equations. If the Mach number in the inviscid flow above such a shear layer is very large (hypersonic), the layer will be very hot and the nondimensional temperature on one or both boundaries, scaled by the peak temperature in the layer, will approach zero in a first approximation. The problem to be considered here, which arises in an asymptotic description of "hypersonic strong viscous interaction" [1] in the presence of large surface mass injection [2], is concerned with a self-similar free shear layer for a limiting case in which the uniform inviscid stream above has infinite Mach number and zero scaled-temperature, while the gas below the layer is at rest, also with zero scaled-temperature. The desired solution has a self-similar form, and so the partial differential equations describing the flow are reduced to a system of ordinary differential equations. The two-point boundary-value problem to be solved is one in which the location of one boundary, at which the solution is singular, is not known a priori, and is determined by imposing boundary conditions which require that the solution have the correct asymptotic behavior as the boundary is approached. Our purpose here is to describe a Newton-based finite-difference method utilizing continuation which was applied successfully to this particular problem. An appropriate choice of continuation path which accounts for the singular nature of the solution is shown, and the behavior of neighboring solutions, which are relevant to hypersonic flow, is illustrated. The numerical formulation may perhaps be useful in solving other boundary-value problems whith similar features.

If a nondimensional streamwise coordinate $x$ and stream function $\psi$ are adopted as independent variables, the boundary-layer equations expressing conservation of momentum and energy for a fluid element may be written respectively as $[1,2]$

$$
\begin{gathered}
u_{x}+\frac{T}{\gamma p u} \frac{d p}{d x}=p\left(T^{\omega-1} u u_{\psi}\right)_{\psi} \\
T_{x}-\frac{(\gamma-1) T}{\gamma p} \frac{d p}{d x}=p\left(T^{\omega-1} u T_{\psi}\right)_{\psi}+(\gamma-1) p T^{\omega-1} u\left(u_{\psi}\right)^{2}
\end{gathered}
$$


where $u, T$, and $p(x)$ are the nondimensional strcamwisc velocity component, temperature, and pressure, respectively; $\gamma$ is the ratio of specific heats (assumed constant); the Prandtl number has been taken equal to one; the viscosity coefficient has been assumed proportional to $T^{\omega}$, with $\frac{1}{2}<\omega<1$; and the gas is assumed to obey the perfect-gas law. The quantities $x, \psi, u, T$, and $p$ are referred to $X_{r}$, $M_{\infty}^{-2} \rho_{\infty} u_{\infty} X_{r}, u_{\infty} M_{\infty}^{2} T_{\infty}$, and $p_{\infty}$, respectively, where $X_{r}$ is a reference length [2], and $M_{\infty}, \rho_{\infty}, u_{\infty}, T_{\infty}$, and $p_{\infty}$ are the Mach number ( $\gg 1$ ), density, speed, temperature, and pressure, respectively, in the undisturbed stream above the layer. For the special case in which the pressure varies as a power of the distance $x$, the solution has a self-similar form. In particular, if the pressure is $p=$ (const) $x^{b}$, the similarity variable is $\zeta=$ (const) $\psi / x^{(1+b) / 2}$ and (1)-(2) are reduced to the ordinary differential equations

$$
\begin{gathered}
\left(T^{\omega-1} u u^{\prime}\right)^{\prime}+\frac{(1+b)}{2} \zeta u^{\prime}-\frac{b}{\gamma} \frac{T}{u}=0 \\
\left(T^{\omega-1} u T^{\prime}\right)^{\prime}+\frac{(1+b)}{2} \zeta T^{\prime}+\frac{(\gamma-1) b}{\gamma} T+(\gamma-1) T^{\omega-1} u u^{\prime 2}=0,
\end{gathered}
$$

where $u=u(\zeta)$, and $T=T(\zeta)$; for the case to be considered here [2], $b=-\frac{1}{2}$.

The boundary conditions for (3) and (4) will typically be given at two values of $\zeta$, say $\zeta=\infty$, which in physical space corresponds to the upper "edge" of the viscous layer, and $\zeta=\zeta_{L}$, where the value of $\zeta_{L}$ depends on the problem considered; typically $\zeta_{\mathrm{L}}$ corresponds to the location of a solid surface, in the case of a boundary layer, or to the location of the lower "edge," in the case of a free shear layer. (In the limit of high Mach number, the upper and lower "edges" of the shear layer are located at finite values of the scaled distance coordinate [1,2], as a consequence of the extremely low density of the fluid in the layer and the strong decrease in diffusion near the edges.) For a boundary layer along an impermeable surface, $\zeta_{L}=0$; if the surface is permcable with a suitably small mass flux normal to the wall varying as a particular power of $x$, then $\zeta_{\mathrm{L}}$ is related to the (specified) rate at which mass is added $\left(\zeta_{L}<0\right)$ or removed $\left(\zeta_{L}>0\right)$ at the surface. For the larger mass flux considered here, the viscous boundary layer is "blown away" from the wall as a free shear layer [2], and $\zeta_{\mathrm{L}}=\zeta_{0}$, where $\zeta_{0}$ is an unknown negative constant related to the amount of mass (smaller than the total injected mass) entrained in the lower part of the shear layer. The unusual combination of conditions $u=0$ and $T=0$ at the lower boundary arises because an asymptotic solution is sought in the limit as $M_{\infty} \rightarrow \infty$, and the velocity and temperature are of higher order below the layer in comparison with their values in the layer. To define $\zeta_{0}$ uniquely, however, it is not sufficient simply to specify $u=T=0$ at the lower boundary, since in general there exist infinitely many solutions with arbitrary values of $\zeta_{L}$ which satisfy this condition. (These correspond to a boundary layer with mass transfer at the wall, as mentioned above.) The desired solution may be identified by the way in which $u \rightarrow 0$ and $T \rightarrow 0$ as the lower boundary is approached. Since $u$ and $T$ are zero at 
$\zeta=\zeta_{0}$, the coefficients of $u^{\prime \prime}$ and $T^{\prime \prime}$ in (3) and (4) also vanish at that point. The asymptotic behavior as $\zeta \rightarrow \zeta_{0}$ is given by

$$
\begin{aligned}
& u=\left(\zeta_{0}^{2} \bar{\zeta}^{\omega}\right)^{1 /(2 \omega-1)}\left\{w_{0}+w_{1} \bar{\zeta}+w_{2} \bar{\zeta}^{2}+\cdots\right\} \\
& T=\left(\zeta_{0}^{4} \bar{\zeta}\right)^{1 /(2 \omega-1)}\left\{s_{0}+s_{1} \bar{\zeta}+s_{2} \bar{\zeta}^{2}+\cdots\right\},
\end{aligned}
$$

where $\bar{\zeta}=-\left(\zeta-\zeta_{0}\right) / \zeta_{0}$ and $w_{0}, s_{0}, w_{1}$, etc., are constants which depend on $b, \omega$, and $\gamma$; the convection, pressure-gradient, and diffusion terms are then of the same order in $\zeta-\zeta_{0}$, and the shear stress and heat-transfer rate vanish at the lower boundary, as expected from a physical point of view. If $\omega \rightarrow 1$, a logarithm also appears, whereas if $\omega \rightarrow \frac{1}{2}$, then $\zeta_{0} \rightarrow-\infty$ and the behavior is exponential rather than algebraic. The boundary conditions to be used for (3)-(4) when $\frac{1}{2}<\omega<1$ are then

$$
\begin{array}{lll}
\zeta \rightarrow \zeta_{0}: & u \sim(\text { const })\left(\zeta-\zeta_{0}\right)^{\omega /(2 \omega-1)}, & T \sim(\text { const })\left(\zeta-\zeta_{0}\right)^{1 /(2 \omega-1)} \\
\zeta \rightarrow \infty: & u \rightarrow 1, & T \rightarrow 0 .
\end{array}
$$

Several numerical techniques have been used to solve the self-similar form of the compressible laminar boundary-layer equations, and the need to specify asymptotic boundary conditions for uniqueness has been encountered before in computing boundary layer solutions with $b>0$ (e.g., Ref. [3]). Early solutions with $\zeta_{L}=0$ were computed by Cohen and Reshotko [3] using a successive-approximation scheme. For the case where $\zeta_{L}$ is a large negative number (with $\zeta_{L}$ specified explicitly), shooting from the interior was found successful by Nachtsheim and Green [4] as was the Newton-iteration finite-difference method described by Liu and Chiu [5]. Nachtsheim and Swigert [6] developed a shooting method to handle asymptotic boundary conditions. Initial attempts at solving the present problem using a shooting method combined with the known asymptotic behavior and certain invariance properties of the system were unsuccessful. An approach that was successful is described below.

\section{Numerical Method}

To handle the difficulties at the lower boundary, a continuation method is employed with the location $\zeta_{\mathrm{L}}$ of this boundary treated as a free parameter. ( $\zeta_{0}$ denotes the value of $\zeta_{L}$ for which (7) is satisfied.) To identify a feasible continuation path, several trial solutions were computed for various values of $\zeta_{L}$ with $u\left(\zeta_{L}\right)=0$, $T\left(\zeta_{\mathrm{L}}\right)=T_{\mathrm{L}}, u(24)=1$, and $T(24)=0$, where $0 \leqslant T_{\mathrm{L}} \ll 1$. It was found that solutions with $\zeta_{\mathrm{L}}<\zeta_{0}$ and $T_{\mathrm{L}}=0$ could not be computed directly since a discontinuity in the behavior of the solution appears at $\zeta_{0}$ : the trivial solution $u=T=0$ occurs for $\zeta<\zeta_{0}$, and the desired fractional-power behavior (7) occurs for $0<\zeta-\zeta_{0} \ll 1$. Thus, for any $\omega$ in the specified range $\frac{1}{2}<\omega<1$, derivatives of $u$ and $T$ of sufficiently high order are discontinuous at $\zeta=\zeta_{0}$, and one may expect computational difficulties if 

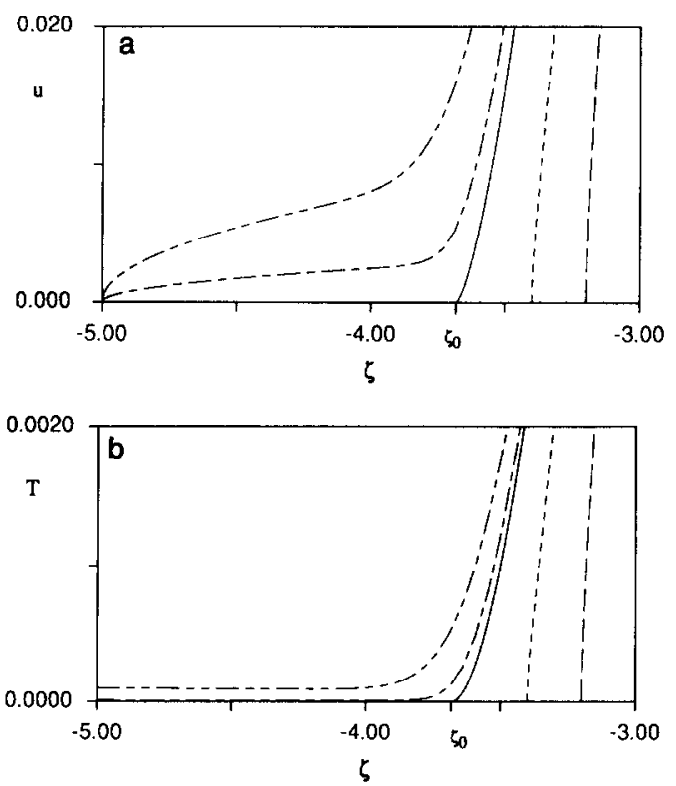

FIG. 1. Trial numerical solutions (broken lines) and the desired solution (solid line) for $u$ (a) and $T$ (b) near $\zeta=\zeta_{0}$. With $T_{\mathrm{L}}=0$ : $\zeta_{\mathrm{L}}=-3.2(--)$; $\zeta_{\mathrm{L}}=-3.4(---)$. With $\zeta_{\mathrm{L}}=-5: T_{\mathrm{L}}=10^{-4}$ ( ); $T_{\mathrm{L}}=10^{-5}(\quad)$.

an assumed location for the lower boundary in some iterative numerical scheme is less than $\zeta_{0}$ (that is, if $\zeta_{0}$ lies within the computational domain). Figure 1 provides an illustration of such behavior for the case $\gamma=1.4, \omega=\frac{3}{4}$, and $b=-\frac{1}{2}$. The two curves to the left are typical of solutions with $T_{\mathrm{L}}>0, \zeta_{\mathrm{L}}<\zeta_{0}$, and the two curves to the right are typical of solutions with $T_{\mathrm{L}}=0, \zeta_{\mathrm{L}}>\zeta_{0}$; note that as $T_{\mathrm{L}} \rightarrow 0, u \rightarrow 0$ and $T \rightarrow 0$ for $\zeta_{\mathrm{L}}<\zeta<\zeta_{0}$.

The problem is now reformulated as follows. The new independent variable

$$
x \equiv \zeta-\zeta_{\mathrm{L}}
$$

is introduced, and solutions are considered on the truncated domain $0 \leqslant x \leqslant x_{\mathrm{U}}$, where $x_{\mathrm{U}}$ is chosen large enough that (8) is satisfied within a specified tolerance (both $1-u$ and $T$ are $O\left(x^{-2 /(1-w)}\right)$ as $\left.x \rightarrow \infty\right)$. The system (3)-(4) will be written in terms of $x$ as a two-element vector equation in the form

$$
\mathbf{r}\left(\mathbf{s} ; \zeta_{\mathrm{L}}\right)=\mathbf{0}
$$

where $\mathbf{s}^{\mathbf{T}} \equiv\left(u\left(x ; \zeta_{\mathbf{L}}\right), T\left(x ; \zeta_{\mathrm{L}}\right)\right)$ and $\mathbf{r}$ is the appropriate vector differential expression. The boundary conditions

$$
\begin{array}{lll}
x=0: & u=0, & T=0 \\
x=x_{\mathrm{U}}: & u=1, & T=0
\end{array}
$$


arc now adopted, together with the additional condition

$$
\left.g\left(\zeta_{\mathrm{L}}\right)\right|_{\zeta_{\mathrm{L}}=\zeta_{0}}=0 \text {, }
$$

where $g$ is a function which measures the agreement between the numerical and asymptotic solutions near the lower boundary, vanishing when (7) is satisfied. (The form for $g$ is not unique; a convenient choice will be described later.) Starting with an initial guess $\zeta_{\mathrm{L}}^{(0)}>\zeta_{0}$, the numerical procedure is to march in $\zeta_{\mathrm{L}}$, solving (10)-(12) at each step and approaching $\zeta_{0}$ from above so that the singularity at $\zeta_{0}$ lies outside the computational domain. Marching is terminated when (13) is satisfied within some desired level of accuracy.

To solve the boundary-value problem (10)-(12) at each iteration level in $\zeta_{\mathbf{L}}$, a Newton-based method is adopted, utilizing second-order finite difference and a nonuniform computational grid. On the basis of the first two terms in the asymptotic expansions for $u$ and $T$ as $\zeta \rightarrow \zeta_{0}$, it is found that (7) is an accurate representation of the solution with maximum fractional error of order $10^{-3}$ over the small interval $0<\zeta-\zeta_{0} \leqslant \varepsilon_{a}$, where $\varepsilon_{a}$ is of order $10^{-3}$ for $\omega=\frac{3}{4}$. To resolve the solution in this small region without requiring an excessive number of grid points, the "exponential" grid

$$
x_{j}=x_{0}+\frac{\left(\alpha^{j}-1\right)}{(\alpha-1)} \Delta x_{0}, \quad j=1, \ldots, N,
$$

is employed, where $x_{0}=0 ; \Delta x_{0} \equiv x_{1}-x_{0}$ is specified (typically of order $10^{-4}$ to $\left.10^{-3}\right)$; and the constant $\alpha \equiv\left(x_{j+1}-x_{j}\right) /\left(x_{j}-x_{j-1}\right)>1 \quad(\approx 1.03$, for example $)$ satisfies

$$
\frac{\alpha^{N}-1}{\alpha-1}=\frac{x_{N}}{\Delta x_{0}}
$$

with $x_{N}=x_{\mathrm{U}}$ specified $(\approx 24$, typically).

The system (10) may be written in a form suitable for numerical solution: the diffusion terms are expanded and all negative powers of $u$ and $T$ are factored away to avoid possible floating-point overflow during iteration and inaccuracies associated with computing ratios of terms that vanish near $\zeta_{0}$; first and second derivatives are then replaced with three-point central-difference formulas appropriate for the grid (14), e.g.,

$$
\begin{gathered}
d u /\left.d x\right|_{x=x_{j}} \approx u_{x_{j}} \equiv \frac{u_{j+1}+\left(\alpha^{2}-1\right) u_{j}-\alpha^{2} u_{j-1}}{\Delta x_{j-1} \alpha(1+\alpha)} \\
d^{2} u /\left.d x^{2}\right|_{x=x_{j}} \approx u_{x x_{j}} \equiv \frac{u_{j+1}-(\alpha+1) u_{j}+\alpha u_{j-1}}{\left(\Delta x_{j-1}\right)^{2} \alpha(1+\alpha) / 2},
\end{gathered}
$$

where $\Delta x_{j-1} \equiv x_{j}-x_{j-1}$. The errors are $O\left(\left(\Delta x_{j}\right)^{2}\right)$ in (16), and if $\alpha=1+O\left(\Delta x_{j}\right)$, in (17) as well. The numerical approximation to system (10) thus reads

$$
\mathbf{r}_{j}=\mathbf{r}_{j}\left(\mathbf{s}_{j-1}, \mathbf{s}_{j}, \mathbf{s}_{j+1} ; \zeta_{\mathrm{L}}\right)=O\left(\left(\Delta x_{j}\right)^{2}\right),
$$


where the first element of $\mathbf{r}_{j}$ is given by

$$
r_{1_{j}} \equiv T_{j} u_{j}^{2} u_{x x_{j}}+\left\{(\omega-1) T_{x_{j}} u_{j}+T_{j} u_{x_{j}}+\frac{(1+b)}{2}\left(x_{j}+\zeta_{\mathrm{L}}\right) T_{j}^{(2-\omega)}\right\} u_{j} u_{x_{j}}-\frac{b}{\gamma} T_{j}^{(3-\omega)}
$$

and the second by

$$
\begin{aligned}
r_{2,} \equiv & T_{j} u_{j} T_{x x_{j}}+\left\{(\omega-1) T_{x_{j}} u_{j}+T_{j} u_{x_{j}}+\frac{(1+b)}{2}\left(x_{j}+\zeta_{L}\right) T_{j}^{(2-\omega)}\right\} T_{x_{j}} \\
& +\frac{(\gamma-1) b}{\gamma} T_{j}^{(3-\omega)}+(\gamma-1) T_{j} u_{j} u_{x_{j}}^{2}
\end{aligned}
$$

with the subscript $j$ denoting evaluation at $x_{j}$. Requiring (18) to vanish at the $N-1$ interior grid points and using the boundary conditions (11)-(12), one obtains the nonlinear algebraic system

$$
\begin{gathered}
\mathbf{R}\left(\mathbf{S} ; \zeta_{\mathrm{L}}\right)=\mathbf{0} \\
\mathbf{s}_{0}^{\mathrm{T}}=(0,0), \quad \mathbf{s}_{N}^{\mathrm{T}}=(1,0),
\end{gathered}
$$

where $\mathbf{S}^{\mathrm{T}}=\mathbf{S}^{\mathrm{T}}\left(\zeta_{\mathrm{L}}\right) \equiv\left(\mathbf{s}_{1}, \mathbf{s}_{2}, \ldots, \mathbf{s}_{N-1}\right)$, and the "residual vector" $\mathbf{R}^{\mathrm{T}} \equiv\left(\mathbf{r}_{1}, \mathbf{r}_{2}, \ldots, \mathbf{r}_{N-1}\right)$.

Given a value of $\zeta_{L}$ and an initial distribution $\mathbf{S}^{(0)}$, the system (21)-(22) may be solved using a variant of Newton's method which reads at the $k$ th iteration level (as denoted by superscript $k$ )

$$
\begin{aligned}
(\partial \mathbf{R} / \partial \mathbf{S})^{(k)} \Delta^{(k)} & =-\mathbf{R}^{(k)} \\
\mathbf{S}^{(k+1)} & =\mathbf{S}^{(k)}+\sigma_{1}^{(k)} \Delta^{(k)},
\end{aligned}
$$

where $(\partial \mathbf{R} / \partial \mathbf{S})^{(k)}$ is the $2(N-1) \times 2(N-1)$ Jacobian matrix associated with $\mathbf{R}^{(k)}$, and $\Delta^{(k)}$ is the solution of the linear system (23) (the estimated required change in $\mathbf{S}^{(k)}$ to give $\mathbf{R}^{(k+1)}=\mathbf{0}$ ). The "relaxation factor" $\sigma_{1}^{(k)}$ is included in (24) to limit the maximum fractional change in any element of $\mathbf{S}^{(k)}$ over one iteration cycle, a device which aids convergence when the $k$ th iterate is not sufficiently close to the correct solution; the factor is defined by

$$
\sigma_{i}^{(k)}=\min \left(1, \delta / \max _{i}\left|\Delta_{i}^{(k)} / S_{i}^{(k)}\right|\right)
$$

where $\delta$ is a specified constant ( $\approx 0.3$, typically), and $\Delta_{i}^{(k)}$ and $S_{i}^{(k)}$ are the $i$ th elements of $\boldsymbol{\Delta}^{(k)}$ and $\mathbf{S}^{(k)}$, respectively. The iterate $\mathbf{S}^{(k)}$ is considered converged when

$$
\max \left|\Delta_{i}^{(k+1)} / S_{i}^{(k+1)}\right| \leqslant \varepsilon_{1} \ll 1,
$$


where $\varepsilon_{1}$ is specified (typically $\approx 10^{-4}$ ). The matrix $\partial \mathbf{R} / \partial \mathbf{S}$ is of block-tridiagonal form with the $j$ th $2 \times 2$ subdiagonal, diagonal, and superdiagonal blocks given by $\partial \mathbf{r}_{j} / \partial \mathbf{s}_{j-1}, \partial \mathbf{r}_{j} / \partial \mathbf{s}_{j}$, and $\partial \mathbf{r}_{j} / \partial \mathbf{s}_{j+1}$, respectively; system (23) may thus be solved efficiently using a routine such as that listed in [7].

Given the start-up values $\zeta_{\mathrm{L}}^{(0)}$ and $\zeta_{\mathrm{L}}^{(1)}$ (both $>\zeta_{0}$, e.g., 0.01 and 0$)$, the $(m+1)$ th iterate $\zeta_{\mathrm{L}}^{(m+1)}$ is computed using a variant of the secant method

$$
\zeta_{\mathrm{L}}^{(m+1)}=\zeta_{\mathrm{L}}^{(m)}-\sigma_{2}^{(m)} \frac{g^{(m)}}{\left(g^{(m)}-g^{(m-1)}\right) /\left(\zeta_{\mathrm{L}}^{(m)}-\zeta_{\mathrm{L}}^{(m-1)}\right)},
$$

where $g^{(m)} \equiv g\left(\zeta_{\mathrm{L}}^{(m)}\right)$ is evaluated from $\mathbf{S}\left(\zeta_{\mathrm{L}}^{(m)}\right)$, the solution of (21)-(22) computed by solving (23)-(24) with $\zeta_{\mathrm{L}}=\zeta_{\mathrm{L}}^{(m)}$ and the initial distribution $\mathbf{S}\left(\zeta_{\mathrm{L}}^{(m-1)}\right)$. The relaxation factor, $0<\sigma_{2}^{(m)}<1$, is included to avoid overshooting $\zeta_{0}$ and is adjusted during marching based on the number of iterations required to solve (21)-(22); specifically, if (27) gives a value of $\zeta_{\mathrm{L}}^{(m+1)}$ for which (23)-(24) does not converge within a specified number of iterations, $\sigma_{2}^{(m)}$ is replaced with a lower value such as $\left(\sigma_{2}^{(m)}\right)^{2},(27)$ is then reevaluated, and the $(m+1)$ th iteration restarted. It is found numerically that (23)-(24) will not converge in the sense of (26) when $\zeta_{\mathrm{L}}<\zeta_{0}$; thus the inclusion of $\sigma_{2}^{(m)}$ in (27) is a necessity. The iterate $\zeta_{\mathrm{L}}^{(m)}$ is considered converged when

$$
\left|\zeta_{\mathrm{L}}^{(m+1)}-\zeta_{\mathrm{L}}^{(m)}\right| /\left|\zeta_{\mathrm{L}}^{(m)}\right| \leqslant \varepsilon_{2} \ll 1,
$$

where $\varepsilon_{2}$ is specified (typically $\approx 10^{-5}$ ).

To compute $\mathbf{S}\left(\zeta_{\mathrm{L}}^{(0)}\right)$, (23)-(24) may be solved starting with the simple piecewise linear distributions $u=x / 6$ for $x \leqslant 6, u=1$ for $x \geqslant 6$, and $T=T_{\mathrm{L}}\left(1-x / x_{\mathrm{U}}\right)$; continuation is then applied as the lower boundary temperature $T_{\mathrm{L}}$ is incrementally reduced from an initial value 0.02 (say) to 0 , thus providing an initial distribution to begin marching in $\zeta_{L}$.

The choice of the function $g$ is arbitrary and need only provide a sufficient condition for satisfying (7). One might, for example, select $g\left(\zeta_{\mathrm{L}}\right)=d E / d \zeta_{\mathrm{L}}$, where $E$ is the square of the $L_{2}$-norm of the relative error between the asymptotic and numerical solution evaluated over the small interval $0 \leqslant x \leqslant \varepsilon_{a} ; g$ vanishes when $E$ is a minimum, i.e., when $\zeta_{L}=\zeta_{0}$. Since (23)-(24) will not converge when $\zeta_{L}<\zeta_{0}$, one may define a simpler criterion on $\zeta_{L} \geqslant \zeta_{0}$ alone. A possibility which works well for $\omega$ near $\frac{3}{4}$ is

$$
g\left(\zeta_{\mathrm{L}}\right) \equiv \frac{T_{1}(1+\alpha)^{2}-T_{2}}{\Delta x_{0} \alpha(1+\alpha)}, \quad \zeta_{\mathrm{L}} \geqslant \zeta_{0}
$$

which is a second-order forward-difference representation of $d T / d x\left(0 ; \zeta_{L}\right)$ with $T_{0}=0$; this expression is particularly well suited for the case $\omega=\frac{3}{4}$, since (7) gives $T=O\left(x^{2}\right)$ for $0 \leqslant x \ll 1$ when $\zeta_{\mathrm{L}}=\zeta_{0}$, and such quadratic behavior may be computed exactly by $(29)$. 


\section{RESUlts}

A typical solution computed using (29) is illustrated in Fig. 2 for $\gamma=1.4, \omega=\frac{3}{4}$, $b=-\frac{1}{2}, N=200, \Delta x_{0}=10^{-3}$, and $x_{U}=24(\alpha \approx 1.034)$. Both $u$ and $T$ versus $x$ are plotted with most of the computational domain shown in part (a), and a blowup of the region $0 \leqslant x \leqslant 0.02$ shown in part (b). For comparison, the second-order asymptotic solutions (5)-(6) are also plotted in part (b), where relative errors between the numerical and the asymptotic solutions are seen to be of order $2 \%$ for $0 \leqslant x \leqslant 0.01$. (The values of the constants appearing in (5) and (6) for this case are $w_{0}=0.128087, w_{1}=-0.0969801, w_{2}=0.022671, s_{0}=0.00430664, s_{1}=-0.00500391$, $s_{2}=0.00577530$.) The good agreement provides a partial validation of the numerical results. For the case shown, solutions computed with larger $N$ indicate that $N=200$, (which required approximately 3 min to compute on an Apollo DN 4000) gives an accuracy of approximately four significant figures over regions where the solution is of order 1 , falling to approximately two significant figures near $x=0$; the computed location of the lower boundary, $\zeta_{0}=-3.683$, is believed to be accurate to within less than $0.1 \%$.

The rather large value of $N$ required for reasonable accuracy, even with the use of grid (14), is dictated by the need to resolve the solution near $x=0$. When the grid was overly stretched ( $\alpha$ too large), accuracy was degraded and it proved difficult to obtain converged solutions; the allowable amount of stretch is therefore
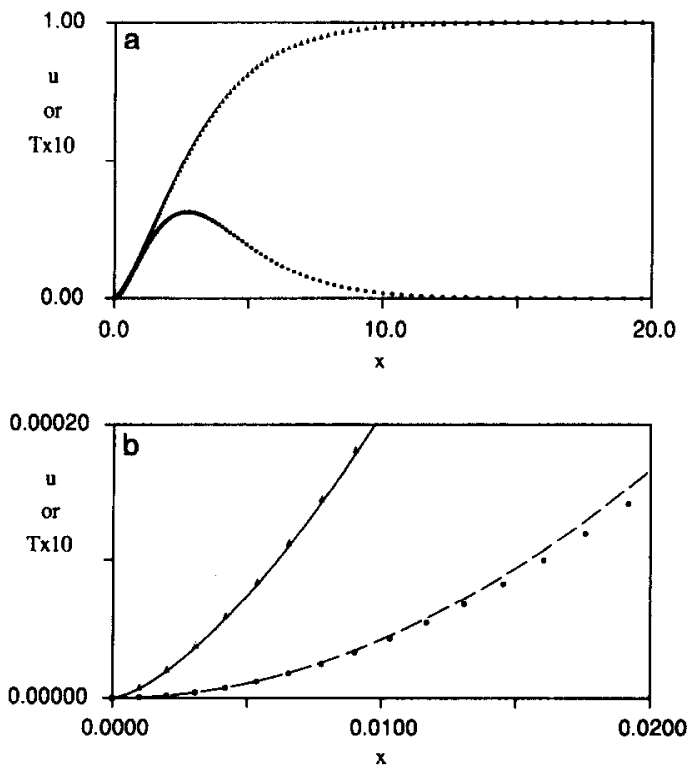

Fig. 2. (a) Profiles of $u(\Delta)$ and $T \times 10(\bullet)$; (b) Comparison with sccond-order asymptotic solutions for $u(-)$ and $T(-\longrightarrow)$ near $x=0$. 
limited and since $\Delta x_{0}$ must be quite small, $N$ must be large. If $\zeta_{0}$ were known a priori, then the required resolution near $x=0$ would not be as great, and $N \approx 50$ with the same $x_{\mathrm{U}}$ and $\alpha$ (but larger $\Delta x_{0}$ ) would have provided an equivalent level of accuracy over most of the grid for the case illustrated in Fig. 2.

The replacement of asymptotic boundary conditions with numerical boundary conditions plus a sufficient condition $g=0$, e.g., the replacement of (7) with (11) plus (29), appears to offer a useful general technique. In the example presented here, a particularly simple function $g$ could be defined since the behavior of neighboring solutions was well understood after computation of several trial solutions. Possibly the general case might best be handled with $g$ based on some least-squares error measure, similar to the ideas presented in [6].

\section{ACKNOWLEDGMENTS}

Thanks are extended to Professor Bram van Leer and Professor Thomas C. Adamson, Jr., for their helpful assistance. This work was supported in part by the U.S. Army Strategic Defense Command.

\section{REFERENCES}

1. K. Stewartson, The Theory of Laminar Boundary Layers in Compressible Fluids (Oxford, London, 1964).

2. A. F. Messiter and M. D. Matarrese, J. Fluid Mech. 219, 291 (1990).

3. C. B. Cohien and E. Reshotko, NACA Rep. 1293 (1956).

4. P. R. Nachtsheim and M. J. GreEN, AlAA J. 9,533 (1971).

5. T.-M. LiU AND H. H. ChIU, $A I A A$ J. 14, (1976).

6. P. R. NACHTSHEIM AND P. SWIGERT, NASA TN D-3004 (1965).

7. D. A. Anderson, J. C. Tannehill, and R. H. Pletcher, Computational Fluid Mechanics and Heat Transfer (Hemisphere, Washington, 1984), p. 551

M. D. MatarRese

A. F. MESSITER

Department of Aerospace Engineering

University of Michigan

Ann Arbor, Michigan 48109-2140 